\title{
Theoretical Research in Elementary Particle Physics
}

This report covers the final period, August 1971 through March 1972, of our research activity under the AEC contract AT(11-1)-264

P.G.O. FREUND's main interests are centered on two subjects: properties of high energy scattering processes, and models of leptonic and hadronic symmetries.

He had earlier introduced the concept of D spin which formalizes the selection rules known in the quark model interpretation of duality. This paper has now been published. ${ }^{1}$ In another paper, he describes a method for incorporating quark spin in the duai resonance mode1. ${ }^{2}$

The role of tensor mesons in high energy scattering is discussed in three papers. First ${ }^{3}$ the phenomenological consequences of the tensor meson dominance in the energy-momentum tensor are explored. Next ${ }^{4}$ it is pointed out that the existence of exotic tensor mesons would remove a normalization difficulty in the matrix elements of the energy-momentum tensor. Regarding the nature of the Pomeranchuk term, the two-component theory of duality. suggests its $f$-dominance. Tests of $f$-dominance in diffraction dissociation are considered and the so-called Morrison rule is shown to be incompatible with f-dominance as well as with experiment. ${ }^{5}$

In three papers ${ }^{6,7,8}$ the various possibilities for a unified description of leptons and hadrons are explored, extending the basic ideas of Weinberg which deal with the electromagnetic and weak interactions of leptons. The main problem is finding the proper symmetry group upon which a gauge theory is to be built. Special attention is given to the case of chiral $U(4) \times U(4)$ group.

\begin{tabular}{|l|} 
This report was prepared as an account of work \\
sponsored by the United States Government. Neither \\
the United States nor the United States Atomic Energy \\
Commission, nor any of their employees, nor any of \\
their contractors, subcontractors, or their employees, \\
makes any warranty, express or implied, or assumes any \\
legal liability or responsibility for the accuracy, com- \\
pleteness or usefulness of any information, apparatus, \\
product or process disclosed, or represents that its use \\
would not infringe privately owned rights. \\
\hline
\end{tabular}




\section{DISCLAIMER}

This report was prepared as an account of work sponsored by an agency of the United States Government. Neither the United States Government nor any agency Thereof, nor any of their employees, makes any warranty, express or implied, or assumes any legal liability or responsibility for the accuracy, completeness, or usefulness of any information, apparatus, product, or process disclosed, or represents that its use would not infringe privately owned rights. Reference herein to any specific commercial product, process, or service by trade name, trademark, manufacturer, or otherwise does not necessarily constitute or imply its endorsement, recommendation, or favoring by the United States Government or any agency thereof. The views and opinions of authors expressed herein do not necessarily state or reflect those of the United States Government or any agency thereof. 


\section{DISCLAIMER}

Portions of this document may be illegible in electronic image products. Images are produced from the best available original document. 
$\therefore \quad$ In A. Ferber's work, ${ }^{9}$ fits to high energy $\pi N$ differential and total cross sections are made in terms of Regge poles and cuts to learn more about the Pomeranchuk term.

H. C. Tze (actual supervisor Dr. S. Fenster, ANL) is working on a thes is in which dual resonance models of the Virasoro-Shapiro and DrummondRebbi types with vector currents are investigated.

Y. NAMBU has been working on electromagnetic currents in the dual resonance model. His published paper ${ }^{10}$ deals with a general prescription for writing down conserved currents and an approximate dual theory with scaling properties.

With $\mathrm{J}$. Willemsen, he is trying to construct a completely dual model of off-shell electromagnetic amplitudes along the lines of the DrummondRebbi model. A way has been found to remove the bad Gaussian behavior of form factors.

With F. Mansouri, he has published a paper ${ }^{12}$ on a geometrical interpretation of the gauge conditions in the dual resonance model. It simplifies the earlier work of Chang and Mansouri (see below) and further generalizes the argument to the case of electromagnetic currents. As a result an amusing condition on the maximum admissible strength of electromagnetic fields is obtained.

A. Hacinliyan has analyzed in some detail the deep inelastic e-p and e-n scattering in terms of the dual model combined with phenomenological assumptions. 13 In addition to getting a fairly satisfactory result, he raises a question regarding the scaling of experimental data on the $W_{1}$ function. 
$\therefore \quad$ G. Barry has completed his thesis work. ${ }^{14}$ It addresses itself to the strange energy dependence of cross sections for $p+p \rightarrow \pi+d$ and $p+p \rightarrow p+d$. He resolves the puzzle by showing that it is due to the composite nature of the deuteron, and he is further able to account for the behavior of backward p-d elastic scattering.

T. Bell is examining ${ }^{15}$ the problem of mu-mesic atoms for which some disagreement between theory and experiment has been reported recentiy by the group of $H$. Anderson at Chicago. Although the origin of the discrepancy is not understood yet, he has found some errors in earlier theoretical work of others. He has also succeeded in computing a correction term to the Krolt-Wichmann formula.

R. OEHME continued to work on high energy scattering amplitudes 16,17 in particular complex Regge poles and cuts in diffraction scattering. ${ }^{18,19}$ He obtains a connection between the impact parameter representation and the Regge formulation with certain consistency requirements. He also shows 20 that a rising total cross section requires the leading trajectory to behave like $\alpha(t) \sim 1+c \sqrt{t}+.$.

In another line of work he has been studying the problem of second class currents in weak interactions, motivated by the Chicago - ANL experiment on $\Lambda$ beta decay. In a recent paper ${ }^{21}$ he discusses the properties of pseudotensor terms generated by either genuine second class currents or a SU(3) breaking in the first class currents. He is also re-examining the question of the origin of the Cabibbo angle.

A Garcia's thesis paper 22 is devoted to a detailed analys is of the $\Lambda$ beta decay data, confirming an earlier claim that a large pseudotensor term is required for its theoretical interpretation. 


\section{Friedman's thesis paper 23 deals with a model of diffraction} scattering which invokes the mechanism of fixed poles and shielding cuts.

R. G. SACHS. The photoproduction of vector mesons in $e^{+} e^{-}$collisions and from nuclei has been analyzed in the $\rho-\omega$ interference region in a model-independent treatment, in collaboration with his student $\mathrm{J}$. L. Lemke. ${ }^{24}$ This enables one to determine three complex parameters from the data, and thereby test various theoretical models.

In another article, ${ }^{25}$ he goes through a careful analysis of the role of time reversal invariance in $k^{\circ}$ meson experiments, and demonstrates the qualitative features of the physically relevant parameters bearing on T-invariance.

Sachs is also one of the co-authors of the Report of the Elementary Particle Physics Panel to the Physics Survey Committee, to be published by the National Academy of Sciences. ${ }^{26}$

W-K. TUNG's work (in collaboration with C. Carison) is mainly concerned with problems of analyzing and carrying out high energy experiments with electron beams in order to extract relevant physical information. In one paper $^{27}$ he discusses the importance and practicability of measuring spindependent structure functions in electron-nucleon (and muon-nucleon) collisions. He obtains bounds on these functions from known structure functions, and predictions from various theoretical models are contrasted: with each other.

The possibility of learning strong interactions from hadron production in $\mathrm{e}^{+} \mathrm{e}^{-}$colliding beam experiments is the subject of another work. Here the most important inclusive and exclusive reactions in two-photon exchange processes are studied. $^{28}$ In particular, the problem of extracting S-wave $\pi-\pi$ scattering phase shift is analyzed in detail. 29 
K. Fujikawa's paper ${ }^{30}$ on a detailed analysis of the weak leptonic processes $\nu+(Z) \rightarrow \nu+\mu+\bar{\mu}+(Z)$ and $\bar{\mu}+(Z) \rightarrow \bar{\mu}+\nu+\bar{\nu}+(Z)$ has been published. He has also carried out calculations 37 on muon pair creation via the two-photon exchange mechanism in charged particle collisions. The result shows an interesting scaling behavior, and is an order of magnitude larger than that due to the ordinary deep inelastic process on photon target. He further discusses the effect of bremsstrahlung processes, and makes a numerical analysis of cross sections.

In another published work ${ }^{32}$ he proposes a simple picture of the scaling of deep inelastic electron-proton scattering based on generalized vector meson dominance. It shows that a very weak spectral function in the large photon mass spectral region is sufficient to give the desired structure functions .

In a paper ${ }^{33}$ written in collaboration with L.N. Chang, ambiguities in applying the current algebra sum rules to single particle inclusive processes, W-production, etc., are pointed out. They are related to the freedom of subtraction terms in the dispersion relation in a sub. energy variable.

L.N. Chang and F. Mansouri ${ }^{34}$ have given a geometrical interpretation to the gauge conditions found in the dual resonance model. These conditions. turn out to mean that one is dealing with a two-dimensional sheet embedded in space-time and its dynamics depends only on the geometry of such a sheet, in a way reminiscent of general relativity.

In a paper by L. N. Chang and N. P. Chang ${ }^{*}$ the canonical Hamiltonian approach to field theory is applied to the high energy behavior of massive quantum electrodynamics. They obtain a formula for the $S$-matrix in which the leading eikonal factor and a correction factor are separated.

${ }^{\star}$ City College of the City University of New York. 
Bibitiography of Works Carried Out Under

Contract No. AT (11-1)-264

for the period August 1971 through March 1972

COO No.

1. P.G.0. Freund, The Three Components of D Spin, Nucl. Phys. B29 317 (1971).

2. P.G.0. Freund, Quark Spin in a Dual Resonance Model, Nuovo Cim., 8 A, 525 (1972).

3. P.G.0. Freund, Tensor Meson Dominance and Regge Couplings, Nuovo Cim., 5A, 9 (7971).

4. P.G.O. Freund, Exotic Mesons and the Hadronic Energy Momentum Tensor, Phys. Lett. 35B, 317 (1971).

5. P.G.O. Freund, H.F. Jones \& R.J. Rivers, Dynamics versus Selection Rules in Diffraction Scattering, Phys. Lett. $\underline{36 \mathrm{~B}}, 289$ (1971).

6. P.G.0. Freund, Leptonic and Hadronic Symmetries, Nuc. Phys. B. (in press).

7. P.G.0. Freund and C.E. Carison, The Case for a Quartet Model of Hadrons, Phys. Lett: $\underline{39 B}, 349$ (1972).

8. P.G.0. Freund, Lepton Number Conserving and Non-Conserving Weak Interactions, Nuc. Phys. B. (in press).

9. A. Ferber, Attempt at a Regge-Pole Regge-Cut Discription of High Energy Scattering, Nuovo Cim. 4A, 1 (1971).

9a. H.C. Tze, Thesis, under preparation.

10. Y. Nambu, Electromagnetic Currents in Dual Hadrodynamics, Phys. Rev. D4, 1193 (1971).

11. J. Willemsen, thesis, under preparation.

12. Y. Nambu and F. Mansouri, Gauge Conditions in Dual Resonance Modeis, Phys. Letters, 39B, 375 (1972).

13. A. Hacinliyan, Deep Inelastic Electron-Proton and Electron-Neutron Scattering, Nuovo Cim. 8A, 541, (1972). 
14: G. Barry, Deuteron Stripping Reactions at High Energy, Annals of Physics, (in press).

15. T. Beil, thesis, under preparation.

16. R. Oehme, Complex Angular Momentum, Springer Tracts in Modern Physics, $\underline{57}$, 13 2 (1971).

17. R. Oehme, Duality and Regge Theory, ibid. p. 179.

18. R. Oehme, Complex Negative Signature Trajectories, Phys. Rev. D3, 3217 , (1971).

19. R. Oehme, Complex Regge Trajectories, Phys. Rev. D4, 1485 (1971)

20. R. Oehme, Rising Cross Sections, Springer Tracts in Modern Physics, 61, 109 (1972).

21. R. Oehme, Second Class Currents and Broken SU(3) Symmetry, EFI Preprint, 72-6.

22. A. Garcia, Analys is of Spin Correlations in the Beta Decay of the Lambda Hyperon, Phys. Rev. D3, 2638 (1971).

23. D. Friedman, Model for Diffraction Scattering with Fixed Poles and Shielding Cuts, Nuovo Cim. (in press).

24. R. G. Sachs and J. L. Lemke, Analys is of Vector-Meson-Photoproduction Experiments in the $\rho-\omega$ Interference Region, Phys. Rev. D5, 590 (1972).

25. R. G. Sachs, Time Reversal, Science Magazine 176, 587 (1972) 600

26. R. G. Sachs, J. D. Bjorken, et al., Report of the Elementary Particle Physics Panel to the Physics Survey Comittee, National Academy of Sciences.

27. W-K. Tung, and C.E. Carlson, Spin Dependent Inelastic Electron-Nucleon Scattering, Phys. Rev. D5, 727 (1972).

28. W-K. Tung, and C.E. Carlson, Two Photon Processes in Colliding Beam Experiments, Phys. Rev. D4, 2873 (1971)

29. W-K. Tung, and C.E. Carlson, Measuring S-Wave $\pi-\pi$ Phase Shifts in Colliding Beam Experiments (to be published in Phys. Rev.). 
30. K. Fujikawa, The Self-Coupling of Weak Lepton Currents in High Energy Neutrino and Muon Reactions, Ann. of Physics, 68, 102 (1977).

31. K. Fujikawa, Nuon Pair Creation via Two Photon Exchange in Charged Particle Collisions:

I. Electron Collisions to be published in Nuovo Cim. 588 II. Hadron Colprisions

32. K. Fujikawa, Inelastic Electron-Proton Scattering and Vector Meson Dominance, Phys. Rev. D4, 2794 (1971).

33. K. Fujikawa and L. N. Chang, Current Algebra in Two Particle Basis and Single Particle Inclusive Processes, Phys. Rev. D5, 2041 (1972).

34. L. N. Chang and F. Mansouri, Dynamics Underlying Duality and Gauge Invariance in the Dual Resonance Model, (to be published in the May 15 issue of Phys. Rev.)

35. L. N. Chang and N. P. Chang, A Canonical Approach to High Energy Scattering in Massive Quantum Electrodynamics, to be published in Phys. Rev. 
List of Individuals Supported by Contract AEC AT(11-1)-264

RESEARCH ASSISTANTS

E. Manessis

H.C. Tze

J. Lemke

J. Wit lemsen

C.A. Matyas

C. Saclioglu

Y. Cho

T. Bel1

S. Soong

R. Stern

$Y$. Band

D. Freidman

S. Paranjape

S. Nandi

\section{RESEARCH ASSOCIATES}

C. Carison

K. Fujikawa

F. Mansouri

A. Hacinliyan (Part Time)

G. Barry (Part Time)

\section{FACULTY}

P.G.0. Freund, Associate Professor

Y. Nambu, Professor

R. Oehme, Professor

R. G. Sachs, Professor

$W-K$. Tung, Assistant Professor

\section{SECRETARIES}

L. $\operatorname{Cox}$

J. Reiffel (1/2 Time) 